M.E. LAPOINIE, «Jake, Joshua et Bamey, intellectuels et transfuges: le paradoxal héroïsme des personnages de M. Richler», dans Y.HAMEletM. BOUCHARD(dir),Portraitdel'homme de lettres en héros, @nalyses, hiver2006

\title{
Martine-Emmanuelle LAPOINTE
}

\section{Jake, Joshua et Barney, intellectuels et transfuges : le paradoxal héroïsme des personnages de Mordecai Richler}

Dans le fragment «Antithèse » de Minima Moralia, Adorno décrit la paradoxale situation de celui qui refuse de faire siens les principes de la société bourgeoise : «[c]elui qui a pris ses distances, écrit-il, est aussi empêtré que celui qui est plongé dans des activités [...] Les distances que l'on prend par rapport aux rouages du système représentent un luxe qui n'est possible que comme produit du système lui-même.» (1991, p. 23) Directement concernés par cette remarque, les intellectuels, d'ajouter Adorno, «sont les derniers ennemis des bourgeois et en même temps les derniers bourgeois », car « ils peuvent encore s'offrir le luxe de la pensée, au lieu de se consacrer à la pure et simple reproduction de l'existence matérielle, ils se comportent comme des privilégiés; mais dans la mesure où ils s'en tiennent à la pensée, ils font voir le néant de ce privilège. » (1991, p. 23) Le constat, certes, peut sembler évident. N'a-t-on pas abondamment glosé les limites et les contradictions du rôle de l'intellectuel, de son discours surtout, qui est souvent condamné à reconduire ce qu'il dénonce? Comment, en effet, mettre ses engagements personnels et sa vie intime au service d'une pensée ou de principes relativement abstraits? Comment paroles et actions peuvent-elles coïncider au point de ne jamais se contredire? Comment assumer le poids des responsabilités qu'engage toute prise de parole publique?

Devant ces contradictions, ces "antithèses » pour reprendre le mot d'Adorno, deux réponses ou deux réactions semblent de prime abord s'imposer: résister en se retirant dans une solitude inentamable, refuser absolument de se compromettre, ou accepter de jouer selon les règles du système, $\mathrm{y}$ agir en toute conscience malgré les paradoxes qu'une telle attitude appelle. Choisir la tour d'ivoire ou le salon mondain, l'ermitage ou la cour, la revue d'avant-garde ou le plateau de télé... Être Blanchot ou BHL, Ducharme ou Tremblay... Mais 
M.E. LAPOINIE, «Jake, Joshua et Bamey, intellectuels et transfuges: le paradoxal héroïsme des personnages de M.Richler», dans Y.HAMElet M.BOUCHARD(dir),Portraitdel'homme de lettres en héros, @nalyses, hiver2006

sûrement pas les deux à la fois. Et si l'on ne pouvait accepter de se cantonner à l'une de ces deux stratégies, si l'on était soumis à un constant tiraillement, si l'on n'avait pas de place à tenir faute de savoir exactement où l'on se situe... À l'instar de Mordecai Richler, on aurait pris le parti de tout confondre, de fréquenter la tour et la cour, sans oublier toutefois de passer par les jardins.

Le point de vue de Richler sur l'engagement de l'intellectuel est en effet paradoxal à plusieurs égards. Dans l'essai «Why I write », paru dans Shovelling Trouble, il admet même :

All of us tend to romanticize the world we nearly chose. In my case, academe. [...] I saw myself no longer a perplexed freelancer with an unpredictable income, balancing this magazine assignment, that film job, against the time it would buy me. No sir. Sipping Tio Pepe in the faculty club, snug in my leather winged-arm chair and the company of other disinterested scholars, I would not, given the assurance of a monthly cheque, chat about anything so coarse as money. ${ }^{1}(1972$, p. 17)

L'académisme, si souvent ridiculisé dans les ouvrages de Richler, est présenté ici sous la forme d'une tentation. Il représente le confort et le luxe, permettrait à l'écrivain de s'adonner à des activités improductives, de faire partie d'une sorte d'aristocratie du milieu artistique évoluant en marge de la société de consommation et fonctionnant selon des règles qui lui seraient propres; en somme, de n'être engagé que dans son propre art et, par là même, de se tenir loin des souillures qui à tout moment menacent celui qui vit de sa pensée et de sa plume.

\footnotetext{
${ }^{1}$ Je traduis : « Nous avons tous tendance à idéaliser le monde que nous avons failli choisir. Dans mon cas, l'université. [...] Je ne me voyais plus tel un écrivain indépendant avec un salaire précaire, jonglant avec l'article de magazine et le scénario de film, de manière à acheter du temps. Non, monsieur, sirotant du Tio Pepe au club de la faculté, bien abrité dans mon fauteuil à oreillettes de cuir, appréciant la compagnie d'autres érudits désintéressés, je ne pourrais, avec la garantie d'un chèque mensuel, parler d'une chose aussi triviale que l'argent. »
} 
M.E. LAPOINIE, «Jake, Joshua et Bamey, intellectuels et transfuges: le paradoxal héroïsme des personnages de M. Richler», dans Y.HAMEl et M. BOUCHARD(dir),Portraitdel'homme de lettres en héros, @nalyses, hiver2006

À ce monde fermé et autonome s'oppose, toujours dans le discours de Richler, le marché des biens culturels qui contraint l'écrivain à produire, à écrire pour différents médias dans le désordre et au hasard des offres et des contrats; bref, à jouer le rôle du polygraphe. Richler navigue entre les deux mondes qu'il se plait à confronter : anthologiste, écrivain en résidence (à l'Université Sir George Williams notamment), auteur d'essais sur la culture et sur la littérature, il a aussi publié des articles dans Playboy, dans Life et dans d'autres publications destinées au grand public. Question de brouiller davantage les cartes, il multiplie, dans ses essais comme dans ses romans, les références à la situation paradoxale de l'intellectuel : solitaires reclus en leurs propres fictions, l'écrivain, le cinéaste, le journaliste n'en demeurent pas moins attirés par la foule, souhaitent tout de même connaitre la gloire et plaire aux générations futures. Comme il le remarque dans son essai «Hemingway Set his Own Hours» paru dans le recueil Broadsides, le métier d'écrivain repose sur un curieux mélange, évoque à la fois la solitude créatrice et la gloire :

Muses. Inspiration. But the truth is, everybody I knew in high school who wasn't going to be another Hank Greenberg or Barney Ross or Maurice "The Rocket" Richard was williing to settle for being a writer. As far as we could make out, Hemingway set his own hours. He seemed to go fishing whenever he felt like it. He was on first terms with Ingrid Bergman and Marlene Dietrich. It had to be a good life. ${ }^{2}$ (1990, p. 1)

Ironisant sur la prétendue liberté de l'écrivain - qui ne semble nullement attaché aux circonstances pragmatiques et aux devoirs officiels —, Richler omet temporairement de considérer l'esprit de sérieux que l'on rattache généralement à sa profession. Pourtant,

\footnotetext{
2 Je traduis: «Muses. Inspiration. Mais en vérité, tous ceux qui, à l'école secondaire, ne voulaient pas être un autre Hank Greenberg ou un Barney Ross ou un Maurice "The Rocket" Richard étaient prêts à devenir écrivains. Pour nous, Hemingway disposait de son temps. Il semblait aller pêcher quand il le souhaitait. Il était au mieux avec Ingrid Bergman et Marlene Dietrich. Ce devait être une bonne vie. »
} 
M.E. LAPOINIE, «Jake, Joshua et Bamey, intellectuels et transfuges: le paradoxal héroïsme des personnages de M.Richler», dans Y.HAMEletM.BOuCHARD(dir.),Portraitdel'homme delettres en hérns, @nalyses, hiver2006

l'influence d'Hemingway sur les fictions richleriennes demeure déterminante. $\mathrm{Au}$ fil de l'œuvre, l'écrivain apparaît comme l'incontournable modèle auquel se mesurent Richler et ses délégués textuels; mieux, il devient la figure tutélaire qui souvent influence une conception de l'écriture et de l'engagement traversée, voire modelée, par des idées contradictoires. De fait, le désir d'immortalité, la quête d'un succès qui ne soit pas éphémère, la foi en une possible communion dans et par l'écriture, caractéristiques d'un certain idéalisme, côtoient sans cesse chez Richler une forme de fatalisme diffus, de cynisme étroitement lié aux exigences de la vie contemporaine. Perdus dans un monde dominé par la cupidité et la superficialité - présentes, il va sans dire, dans tous les milieux sociaux -, les intellectuels richleriens n'ont pas de place assignées et se retrouvent, volontairement ou non, décalés, marginalisés, tiraillés entre des envies contradictoires.

Afin de mieux cerner ces tensions, j'analyserai les parcours similaires de trois personnages fictifs. Jake, Joshua et Barney, respectivement mis en scène dans St. Urbain's Horseman (1971), Joshua Then and Now (2001) et Barney's Version (1997), sont tous nés dans le quartier juif du Mile-End au début des années 1930, ont tous vécu la bohème européenne, en Espagne, en France ou en Angleterre, ont tous été animés par de nobles idéaux, qu'il s'agisse de fournir au cinéma canadien ses premiers chefs-d'œuvre, d'écrire l'ouvrage de référence sur la Guerre civile espagnole ou de soutenir des amis artistes, talentueux, pauvres et ingrats. Mais, de plus en plus conscients de la vanité de l'élite cultivée, ils ont tous choisi de s'en éloigner pour fonder leurs familles et connaitre une forme de rédemption par la vie amoureuse et familiale. Alter ego de Richler — qui a d'ailleurs avoué «when I'm not writing I'm a husband and a father of five ${ }^{3}$ » (1972, p. 19) —, ils partagent plusieurs traits : origines modestes et parcours de "self-made men », méfiance à l'égard des institutions culturelles et des groupes, incapacité à réellement intégrer les milieux cultivés (qui néanmoins les fascinent) et préférence pour les refuges excentrés, bars et tavernes enfumés, le plus souvent fréquentés par des ouvriers et des

\footnotetext{
${ }^{3}$ Je traduis : «Quand je n'écris pas, je suis un époux et un père de cinq enfants. »
} 
M.E. LAPOINIE, «Jake, Joshua et Bamey, intellectuels et transfuges: le paradoxal héroïsme des personnages de M.Richler», dans Y.HAMEletM.BOuCHARD(dir.),Portraitdel'homme delettres en hérns, @nalyses, hiver2006

hommes du peuple forts en gueule. Tous trois sont aussi mêlés à des histoires louches, scandales sexuels pour Jake et Joshua, meurtre inexpliqué dans le cas de Barney. Accusés puis acquittés, ils vivent pendant longtemps sous le regard soupçonneux de leur entourage. Transfuges, singuliers justiciers, ils prétendent, qui plus est, révéler les hypocrisies des sociétés trop policées, mais oublient parfois de considérer leurs propres démissions. Antithétiques, les intellectuels richleriens? Certes. Afin de mieux cerner la dialectique de la bravoure et de l'art qui traverse l'œuvre de Richler, j’examinerai les relations qu'entretiennent les personnages de Jake, Joshua et Barney avec deux types opposés de figures tutélaires: le frère idéalisé, souvent plus talentueux ou plus prometteur que nos trois héros, et/ou le modèle négatif, vilain au sens presque classique du terme qui les obsède parfois de manière aiguë. L'analyse de ces relations paradoxales éclairera la conception qu'ils se font de l'engagement de l'artiste et de l'intellectuel et permettra de mieux les situer dans les microcosmes sociaux dessinés par Richler.

\section{Modèles positifs et négatifs}

Avant d'étudier les personnages de Jake, Joshua et Barney, il importe de situer la notion d'héroïsme ou de bravoure dans le contexte de l'œuvre richlerienne. Des aventures espagnoles d'André mises en scène dans The Acrobats (1954) jusqu'au portrait d'une certaine bohême parisienne offert dans Barney's Version, en passant par les mauvais coups des bandes d'amis de la rue Saint-Urbain dépeints dans plusieurs fictions, l'œuvre romanesque de Richler privilégie la fratrie, une amitié entre jeunes hommes ou hommes mûrs fondée sur des rapports presque filiaux. Le héros richlerien, souvent obsédé par le regard d'autrui, se définit presque toujours en se comparant à ses confrères; il les admire ou les envie, les méprise même parfois, mais toujours accorde une valeur à leur jugement, comme si celui-ci pouvait orienter son devenir et en changer le cours. Mais il est une exception à cette logique. Au sein de la fratrie, réelle ou rêvée, se détachent parfois des êtres hors normes et quasi surhumains dont le regard ne se pose jamais ou presque sur les simples héros richleriens. À cette lignée unique appartiennent notamment Joey Hersh, le fameux cavalier de la 
M.E. LAPOINIE, «Jake, Joshua et Bamey, intellectuels et transfuges: le paradoxal héroïsme des personnages de M.Richler», dans Y.HAMElet M.BOUCHARD(dir),Portraitdel'homme de lettres en héros, @nalyses, hiver2006

rue Saint-Urbain, et Solomon Gursky, le riche aventurier aux multiples masques idolâtré par Moses Berger dans Solomon Gursky Was Here. Fuyards, survenants, spectres, ces deux personnages échappent au pouvoir de la communauté, ne sont jamais contraints de vivre selon les lois de la tribu car ils se placent au-dessus de celles-ci. À Joey comme à Solomon sont de plus accordées des qualités épiques, courage, témérité, sens de la justice, polyvalence, attributs ramenant à un idéal d'action dans la Cité.

Sont hérö̈ques ceux qui n'ont pas à se conformer aux normes sociales ordinaires, ceux qui refusent le prosaïsme du réel, préférant, comme l'a écrit Isabelle Daunais à propos du héros traditionnel, «le temps de l'immortalité, c'est-à-dire le temps en dehors de la vie terrestre » (2002, p. 187). Or, à des degrés différents bien sûr, cette vision des choses informe l'œuvre entière de Richler: on ne se mesure pas à des modèles réels, on envie rarement les héros du Reader's Digest, pour reprendre l'une des idées de l'auteur, mais on invente le héros en lui prêtant des desseins exceptionnels. On attribue un caractère d'irréalité à son existence. Ce faisant, on échappe à la cruauté du quotidien et on vit dans l'univers fictif de l'Autre avec un grand A.

Penchons-nous sur notre premier personnage, Jake Hersh, issu de St. Urbain's Horseman. Il poursuit une carrière de cinéaste à Londres, a épousé, au grand désespoir de son père, Nancy — une Goy —, avec qui il mène une existence bourgeoise et confortable. Reniant ses origines, il ne cesse de critiquer son lieu natal qui évoque, selon lui, médiocrité collective et néant culturel : «Earlier, in Montreal, Jake had earnestly assured his troubled relatives that their city was a cultural desert, a colonial pimple, and he was off to nourish himself at the imperial fountainhead. ${ }^{4} »(1989$, p. 191) "Ashamed of his parents' Yiddish accent ${ }^{5} »(1989$, p. 6), désirant à tout prix fuir le quartier de l'enfance, Jake adopte l'attitude du transfuge, souhaite taire ses origines afin de mieux embrasser la vie culturelle trépidante du

\footnotetext{
${ }^{4}$ Je traduis : « Plus tôt, à Montréal, Jake avait assuré à ses parents troublés que leur ville était un désert culturel, une excroissance coloniale. Il la quittait donc pour s'abreuver à la fontaine impériale. »

5 Je traduis : «Gêné par l'accent yiddish de ses parents ».
} 
M.E. LAPOINIE, «Jake, Joshua et Bamey, intellectuels et transfuges: le paradoxal héroïsme des personnages de M. Richler», dans Y.HAMEletM. BOUCHARD(dir),Portraitdel'homme de lettres en héros, @nalyses, hiver2006

«swinging London» : il devient ainsi volontairement autre, choisit une aliénation compensatoire qui lui ouvrira, pense-t-il, les portes des plus prestigieuses maisons de production, qui lui permettra de pénétrer le milieu artistique londonien et de gravir ses échelons. Au fil du roman, l'on comprend que cette entreprise de distanciation, de refoulement pourrait-on dire, ne concerne pas uniquement Montréal, le Mile-End et sa rue Saint-Urbain. Elle permet également à Jake de renier son pays natal : pour lui, le Canada est un «necessity shelter ${ }^{6} »$ (1989, p. 158); Toronto, sa capitale économique, s'avère provinciale, un «farm-club ${ }^{7}$ » étroit (1989, p. 162). La seule solution demeure l'exil.

D'ailleurs, le seul membre de sa famille à trouver grâce à ses yeux, l'insaisissable Cavalier de la rue Saint-Urbain, a pour ainsi dire disparu tel un fuyard impénitent. Vengeur de l'Holocauste, homme aux mille métiers et aux mille talents, Joey Hersh devient pour Jake une figure héroïque, la seule dans un monde dominé par la corruption. Comme le remarque Norman Ravvin, le Cavalier est sans doute le personnage richlerien qui représente le plus éloquemment le désir de vengeance exprimé haut et fort par l'auteur dans certains de ses essais ("The Holocaust and After» notamment). Plutôt que d'essayer de vivre avec le traumatisme de l'après-guerre, Jake reporte sur Joey ses rêves de revanche historique: "Rather than allowing him to come to terms with the Holocaust and Jewish life after the war, Jake's obsession with the Horseman seems only to provide encouragement for his fantasies of threat and revenge, as well as fuel for his urge to see himself as a potential victim»(Ravvin, 1997, p. 42) Dès l'incipit du roman, le Cavalier est présenté comme un idéal de courage et de témérité pourchassant les infâmes nazis :

Jake wondered if the Doktor, given his declining years, slept with his mouth open, slack, or was it (more characteristically, perhaps) always clamped shut? Doesn't matter. In any event, the Horseman would extract the gold fillings from the

\footnotetext{
6 Je traduis : « refuge par défaut ».

${ }^{7}$ Je traduis : « club de fermiers ».
} 
M.E. LAPOINIE, «Jake, Joshua et Bamey, intellectuels et transfuges: le paradoxal héroïsme des personnages de M. Richler», dans Y.HAMEl et M. BOUCHARD(dir),Portraitdel'homme de lettres en héros, @nalyses, hiver 2006

triangular cleft between his upper teeth with pliers. Slowly, Jake thought, coming abruptly awake in sweat. ${ }^{8}$ » $(1989$, p. 11)

La scène de vengeance, digne des pires ou des meilleurs westerns, présente une nette opposition entre le vilain — le docteur Mengele et le Cavalier qui, tel le Golem selon l'hypothèse de David Sheps ${ }^{9}$, est toujours prêt à bondir pour rendre justice. Or, les rêves de vengeance donnant la vedette, non pas au cousin Joey Hersh, mais bien à son double hérö̈que, ponctuent le récit de St. Urbain's Horseman et mettent clairement au jour une conception quasi romanesque de l'engagement et de l'action dans la Cité.

En plus de racheter le roman familial des Hersh, jugé scandaleusement plat par Jake, la figure du Cavalier devient une sorte d'exutoire permettant d'échapper au sort commun. C'est que la vie entière de Jake ressemble à un scénario brodé de clichés, de topö, de stéréotypes.

If, rather than a code of unspoken nonconformities, there was a battery of written tests for intellectual novices, then Jake felt he would have passed top of the latter-day yeshiva class. He had done all the right wrong things, even to marrying a shiksa, voting for the better candidate to this day and, squeezed in a vise between the moral values of two generations, worrying about Arab civil rights in Israel, on the one hand, and kids

\footnotetext{
${ }^{8}$ Je traduis : « Jake se demandait si le Doktor, vu son grand âge, dormait la bouche ouverte, béante, ou était-elle (de façon plus caractéristique sans doute) toujours hermétiquement fermée? Peu importe. Le Cavalier extrairait les plombages dorés de ses dents supérieures avec des pinces. Doucement, pensa Jake, abruptement réveillé, en sueurs. »

${ }^{9}$ David Sheps (1974) soutient en effet que la figure du Golem sert de modèle au Cavalier de la rue Saint-Urbain : «Jake defines Joey as a Golem, but perhaps only as a slightly ironic screen between Joey and himself. Simbolically, he means more than that. But Jake has become too skeptical, too accustomed to disillusion, to articulate exactly what Joey symbolizes for him. » (p. 88) Sheps ajoute, quelques pages plus loin, que « the ambiguities of Joey's ethics and of his relationship to traditional Jewry, like those to Sabbatianism, correspond to Jake's requirements for an heroic model. His messianic deliverer has to be an exile and heretic. » (p. 90).
} 
M.E. LAPOINIE, «Jake, Joshua et Bamey, intellectuels et transfuges: le paradoxal héroïsme des personnages de M.Richler», dans Y.HAMElet M.BOUCHARD(dir),Portraitdel'homme de lettres en héros, @nalyses, hiver2006

having to make do with impurities in their pot, on the other. ${ }^{10}$ (1989, p. 303)

Dans ce parfait canevas, l'intellectuel est présenté comme un être de tiraillement, «squeezed » pour reprendre le mot de Richler, entre des valeurs sociales de gauche, voire des idéaux humanistes qu'il ne peut incarner totalement, et une vie familiale dominée par des considérations pragmatiques. Tout se passe comme si Jake ne pouvait revendiquer honnêtement la place qui est la sienne, comme s'il persistait à souffrir d'un complexe d'infériorité culturelle.

La scène de l'orgie espagnole, anodine mais évocatrice, illustre plus particulièrement le sentiment d'inconfort de Jake. Même s'il souhaite participer à la fête, Jake n'arrive qu'à endosser le rôle du spectateur passif : «Flushed and embarrassed. Then it struck him that this is what they call an orgy, he was taking part, and its spirit soared. This is living, Yankel. Liberated rebel-without-a-cause living [...] Remember this, Jake thought, cherish it, and he felt very ghetto-liberated, very Hemingway. ${ }^{11}$ "(1989, p. 21) Dans ce court passage, le personnage dialogue littéralement avec sa conscience, se dédouble, n'arrivant pas à se détacher de sa culture propre, de ses origines juives - comme en témoigne le simple surnom de Yankel - et des principes qu'on lui a inculqués. Vouées à justifier sa conduite, ses réflexions le trahissent : Jake, de toute évidence, n'est pas de ceux qui passent à l'action sans réfléchir, les "rebel-without-a-cause», les "ghetto-liberated», les doubles d'Hemingway au sens large. Or, s'il envie tant son cousin Joey, c'est parce qu'il a réussi à passer outre, à mépriser totalement -

\footnotetext{
${ }^{10}$ Je traduis : « Si, plutôt que de codes tacites, il eut existé une série de tests écrits destinés aux aspirants intellectuels, Jake les aurait réussis haut la main. Il avait fait toutes les bonnes mauvaises choses, même épouser une Goy, voter pour le meilleur candidat à ce jour et, tiraillé entre les valeurs morales de deux générations distinctes, s'inquiéter pour les droits des Arabes en Israël d'un côté, et pour les besoins des enfants, de l'autre. »

11 Je traduis : «Rouge et embarrassé. Soudain il réalisa qu'il participait à ce qu'ils appellent communément une orgie, et son esprit s'élança vers le ciel. C'est la vie, Yankel. Une vie libérée, animée par la fureur [...]. Il faut garder cela en mémoire, pensa Jake, chérir ce moment, et il se sentit très "ghetto-émancipé", très Hemingway. »
} 
M.E. LAPOINIE, «Jake, Joshua et Bamey, intellectuels et transfuges: le paradoxal héroïsme des personnages de M.Richler», dans Y.HAMElet M.BOUCHARD(dir),Portraitdel'homme de lettres en héros, @nalyses, hiver2006

et non pas de manière superficielle — les lois de la tribu. Pour l'artiste et intellectuel bourgeois qu'est Jake, la réelle bravoure réside donc dans le déni des conventions, dans une marginalité, voire une liminarité, qui condamne à une réelle solitude.

Publié près de dix ans plus tard, Joshua then and Now (1980) met aussi en scène un homme en rupture avec son milieu d'origine et sa généalogie honteuse - son père est un ex-boxeur mafieux et sa mère, une danseuse nue. Joshua Shapiro, né dans le quartier du Mile-End, s'est exilé en Angleterre où il a réussi à conquérir Pauline, une jeune femme issue de la haute bourgeoisie de Westmount. Il est ensuite revenu au pays et est devenu un chroniqueur sportif reconnu, cela même s'il rêvait d'écrire: "The truth was, he had to admit, he was not so much interested in writing as in being a writer. Somebody well known. $^{12} »(2001$, p. 187) À l'instar de Jake, Joshua a lui aussi l'impatience, l'impudence du conquérant, et le désir de faire oublier ses origines modestes; mieux, il souhaite les venger. S'il pénètre les milieux les plus fermés, c'est toujours en demeurant lui-même, "a St.Urbain boy» (2001, p. 444). Aux yeux des proches de Pauline, Joshua sera toujours le Juif un peu rustre, maladroit au tennis, allergique au country club, «an inhibiting presence [...], a Jewy thundercloud ${ }^{13}$ » (2001, p. 43), se posant toujours volontairement à l'extérieur du cercle, refusant par là même d'adopter les codes et les manières de la haute bourgeoisie.

Si Jake se perçoit tel un futur cinéaste de renom, Joshua rêve de venger les révolutionnaires espagnols, voire de venger l'Espagne qui est à la fois « a country of the imagination », " a territory of the heart » et «the first political kiss ${ }^{14} »(2001$, p. 237). Les rêves de bravoure et l'idéalisme de la jeunesse sont ainsi projetés sur ce territoire inconnu qui demeure, à bien des égards, une vue de l'esprit :

\footnotetext{
${ }^{12}$ Je traduis : «La vérité, il devait l'admettre, c'est qu'il était moins intéressé par l'écriture que par le statut d'auteur. Une figure reconnue, respectée. »

${ }^{13}$ Je traduis : «Une présence gênante [...], une tornade juive ».

${ }^{14}$ Je traduis : «le pays imaginaire», «le territoire chéri », «le premier baiser politique ».
} 
M.E. LAPOINIE, «Jake, Joshua et Bamey, intellectuels et transfuges: le paradoxal héroïsme des personnages de M.Richler», dans Y.HAMElet M.BOUCHARD(dir),Portraitdel'homme de lettres en héros, @nalyses, hiver2006

Like Seymour Kaplan, who shared his obsession with Spain, Joshua drifted to sleep dreaming not [...] of striking out the batting order of the New York Yankees, but of Gary Cooper as Robert Jordan, his leg smashed, taking his place behind a machine gun to cover the retreat of Ingrid Bergman, Akin Tamiroff and the other partisans. ${ }^{15}$ (2001, p. 128)

Dans ce fantasme se juxtaposent des références à peine voilées au film For Whom the Bell Tolls (Sam Wood, 1943), inspiré du roman éponyme d'Ernest Hemingway, publié en 1940. Empruntés au cinéma hollywoodien, les clichés de la Guerre civile espagnole respectent une certaine image stéréotypée de l'héroïsme : le héros est seul contre tous, blessé, mais tel le franc-tireur, il réussit tout de même à protéger les siens. Comme l'a montré Paul Warren (1989), la vedettarisation du héros hollywoodien repose en grande partie sur des topoï hérités d'une certaine conception de la culture états-unienne, soit le triomphe de la civilisation sur la sauvagerie, l'individualisme et l'exercice de la volonté. En plaquant cette imagerie convenue sur ses rêves de bravoure, Joshua Shapiro ne peut que trahir son manque de maturité et son incapacité à accepter une réalité non gouvernée par une morale manichéenne.

Si les images sont empruntées au cinéma, la figure tutélaire, elle, provient de la littérature. Considéré ici aussi comme le représentant de la bohême intellectuelle, Ernest Hemingway est également convoqué à plusieurs reprises pour représenter le courage de l'homme d'action. Selon Richler, la génération de Joshua Shapiro aurait même été marquée par l'écrivain au point de souhaiter le dépasser : «proving to themselves and the essential Mr. Hemingway that they did not lack for cojones $^{16} »(2001$, p. 257), le mot espagnol cojones renvoyant ici au sang-froid, au courage, mais aussi au plus trivial « balls! ».

15 Je traduis : «Comme Seymour Kaplan, qui partageait son obsession pour l'Espagne, Joshua s'endormit, ne rêvant guère [...] de renverser la hiérarchie des New York Yankees, mais bien à Gary Cooper incarnant Robert Jordan, sa jambe écrasée, prenant place derrière une mitraillette pour couvrir la retraite d'Ingrid Bergman, d'Akin Tamiroff et des autres partisans. »

16 Je traduis : «prouvant à leurs compères et à l'incontournable M. Hemingway qu'ils ne manquaient pas de cojones ». 
M.E. LAPOINIE, «Jake, Joshua et Bamey, intellectuels et transfuges: le paradoxal héroïsme des personnages de M.Richler», dans Y.HAMElet M.BOUCHARD(dir),Portraitdel'homme de lettres en héros, @nalyses, hiver2006

Joshua ne fait pas que rêver à l'Espagne, il y voyage aussi alors qu'il n'est âgé que de vingt-et-un ans. Il y recueille d'ailleurs les informations qui lui serviront lors de la rédaction de son seul ouvrage sérieux, The Volonteers. Durant ce voyage qui le hantera pendant de nombreuses années, il rencontre le Dr Dr Mueller, qui deviendra son ennemi juré. Joshua le soupçonne d'avoir été un sympathisant du régime nazi, d'être violemment antisémite et d'user de son autorité pour nuire aux citoyens juifs d'Ibiza. Or, on le devine aisément, Joshua se trompe. Au même titre que son Espagne mythifiée, son vilain ressemble à une caricature: "Are you a man or a mouse, Mueller demanded, biting into that ivory cigarette holder. A man or a mouse, he taunted. Man, mouse. And Joshua, wakening, discovered that he was sliding in sweat. ${ }^{17} »(2001$, p. 125) Comme dans St. Urbain's Horseman, le héros vit virtuellement une confrontation avec un cruel docteur allemand. Mais dans Joshua Then and Now, cette confrontation emprunte la forme d'un souvenir, et non d'un pur fantasme de réparation. Ici, la vengeance n'a pas eu lieu, et n'aura pas lieu. Et pourquoi? Car elle aurait été une vaine entreprise. D'une part, l'affront commis par Joshua relève de l'égotisme et de la vanité plus que de la bravoure : il a tout simplement réussi à ravir la belle Monique au Dr Dr Mueller au vu et au su d'un groupe d'officiers. Il transformera pourtant ce geste en acte hérö̈que : «He had taken on a Nazi mano a mano and demonstrated to Mr. Hemingway and himself he did not lack for cojones. ${ }^{18} \gg(2001$, p. 373) D'autre part, le méchant nazi, apprend-on au fil du roman, n'est autre que l'auteur de westerns Gus McCabe. Un écrivain, un fabulateur, mais un vrai écrivain qui a du succès, et non un journaliste qui, comme Joshua, prétend avoir du talent ${ }^{19}$. Pendant la

17 Je traduis : «Êtes-vous un homme ou une souris, Mueller lui demanda, mordant dans son porte-cigarette en ivoire. Un homme ou une souris, railla-t-il. Homme, souris. Et Joshua en se réveillant découvrit qu'il était couvert de sueur. »

${ }^{18}$ Je traduis : « Il avait vaincu un Nazi et démontré à M. Hemingway comme à lui-même qu'il ne manquait pas de cojones. »

${ }^{19}$ Selon Michael Greenstein (1989, p. 159), cet épisode met également au jour un complexe d'infériorité quasi historique: «The entire episode then turns into a parody of Hemingway in which a Jewish-Canadian poses as a tough American challenging European civilization. » 
M.E. LAPOINIE, «Jake, Joshua et Bamey, intellectuels et transfuges: le paradoxal héroïsme des personnages de M. Richler», dans Y.HAMEletM. BOUCHARD(dir),Portraitdel'homme de lettres en héros, @nalyses, hiver2006

guerre, Mueller écrivait des textes de propagande dans les bureaux de l'armée allemande. Jamais il ne s'est battu. «Are you a man or a mouse? ", la question de Mueller ramène Joshua à sa propre lâcheté, à l'échec de sa supposée action hérö̈que. Comble de l'ironie, lorsqu'il retourne à Ibiza plus de vingt ans plus tard, Joshua découvre que sur les ruines de la villa du défunt Mueller, on a construit un complexe immobilier baptisé le Don Quixote Estate. Celui qui rêvait de devenir Hemingway ne fut rien de plus qu'un double de Don Quichotte.

\section{Prétention ou bravoure?}

Contrairement aux romans précédents, la dernière fiction de Richler, Barney's Version (1997), emprunte la forme d'une autobiographie fictive dans laquelle la chronologie des faits et des événements est rarement respectée. Barney Panofsky, narrateur et personnage central du roman, a connu trois vies — peut-être même quatre. À Paris, il a fréquenté un groupe d'écrivains et de peintres, nord-américains pour la plupart. De retour à Montréal, il a voulu rompre avec le milieu artistique pour mieux infiltrer le "Jewish establishment», puis s'est recyclé dans la production de mauvais films destinés à la télévision. Se retrouvant seul après trois mariages successifs, il tente, dans ses mémoires, de dire la vérité sur son passé, vérité malmenée, considère-t-il, par son ennemi juré l'écrivain Terry McIver. Attaché au passé, le roman donne à lire le travail de reconstruction mémorielle, parfois pénible, auquel se livre le narrateur menacé par la maladie d'Alzheimer. La narration même du roman intègre les pertes de contrôle et les trous de mémoire qui affligent Barney. Un appareil de notes infrapaginales, mis en place par Michael Panofsky, le fils de Barney, constitue une sorte de garde-fou, trace littérale de la mort lente du narrateur.

L'exemple de Barney s'impose en contrepoint des deux autres cas analysés, dans la mesure où il témoigne de légères réorientations au sein de l'œuvre et, par là même, d'une réinterprétation de la dialectique de la bravoure et de l'écriture. Et quelles sont ces réorientations? Même férocement drôle par moments, ce roman a tout d'une tragédie, ce qui rompt avec le caractère essentiellement satirique de St. Urbain's Horseman et de Joshua Then and Now. Barney's Version, qui plus est, se 
M.E. LAPOINIE, «Jake, Joshua et Bamey, intellectuels et transfuges: le paradoxal héroïsme des personnages de M.Richler», dans Y.HAMElet M.BOUCHARD(dir),Portraitdel'homme de lettres en héros, @nalyses, hiver2006

présente comme le récit de la perte : perte de la mémoire certes, mais aussi perte de la femme aimée, des ambitions passées, des idéaux, de l'enthousiasme $^{20}$. Seul dans une ville qui lui ressemble de moins en moins, Barney résiste passivement en attendant la fin.

Comme dans les deux autres romans, un être hante les pensées et les cauchemars du personnage principal. Il s'agit, cette fois, de Boogie (de son vrai nom Bernard Moscovitch), le meilleur ami de Barney, disparu dans des circonstances étranges (Barney est accusé du meurtre de son ami, puis acquitté, faute de preuves). À l'instar de Joey Hersh, Boogie a tout du spectre: il échappe aux lois de la communauté, voyage, disparaît puis réapparaît sans prévenir. Parmi les intellectuels et les artistes que Barney a rencontrés à Paris, Boogie demeure la seule et unique référence : «I had lit out the cultural territories, going to Paris, hoping to be enriched by associating with the pure of hearth, the "unacknowledged legislators of the world" and came home determined not to have anything to do with writers or painters again. Except for Boogie. ${ }^{21} »(1997$, p. 182) Le charisme de Boogie est évidemment rehaussé par les regards d'autrui : Barney prétend qu'il était lié à Norman Mailer, William Styron et Billie Holiday, considéré par plusieurs comme l'auteur du « greatest modern American novel yet to be written ${ }^{22} »(1997$, p. 274). Et, ultime consécration: «a letter

\footnotetext{
${ }^{20}$ Selon Pierre Nepveu (2004, p. 68), l'idée de la perte dominerait même l'œuvre entière de Richler : «Sous les rires, les sarcasmes et les sacrilèges de l'auteur de Mon père, ce héros, de L'Apprentissage de Duddy Kravitz et de la Rue SaintUrbain, une perte et un deuil affleurent en effet, et ce n'est pas par hasard que les romans et récits de Richler demeureront, et cela jusqu'aux plus récents tels $L e$ Monde de Barney, parsemés de mots yiddish, yenta, shviz, pisher, a gute neshune, et d'autres termes ou expressions qui sont comme les fragments de l'univers juif ancien à jamais perdu: lambeaux d'une langue morte rescapés grâce à un discours burlesque qui ne cesse de tourner l'identité à la parodie et au ridicule. »

${ }^{21}$ Je traduis : «J'avais investi les territoires culturels en allant à Paris, j'espérais m'enrichir en fréquentant les cœurs purs, "les inavouables justiciers de notre monde”, et je suis revenu chez moi en espérant n'avoir plus jamais rien à faire avec les écrivains et les peintres. Sauf pour Boogie. »

22 Je traduis : «le plus grand roman américain à venir ».
} 
M.E. LAPOINIE, «Jake, Joshua et Bamey, intellectuels et transfuges: le paradoxal héroïsme des personnages de M.Richler», dans Y.HAMElet M.BOUCHARD(dir),Portraitdel'homme de lettres en héros, @nalyses, hiver2006

might turn up from Cuba, addressed to Boogie [...], and it was from Ernest Hemingway. ${ }^{23} »(1997$, p. 274)

Mais l'hérö̈sme de Boogie, au même titre que la vilenie du Dr Dr Mueller, s'avère des plus problématiques. Voyageur attiré par les paradis artificiels, héroïnomane notoire, Boogie n'a jamais réussi à terminer son grand roman. Sa loyauté à l'égard de Barney peut également être considérée suspecte. N'a-t-il pas séduit la deuxième Mme Panofsky? Fait plus important encore: Barney est tout à fait conscient des failles de son ami disparu. En témoigne la double retranscription de l'ultime conversation des deux amis. La première version de cette discussion a été censurée par son auteur. De retour à son chalet des Laurentides, Barney surprend son meilleur ami au lit avec sa femme; s'ensuivent discussion passionnée et virile beuverie. Pour rire, Barney menace Boogie avec un revolver. La scène se termine sur un échange de plaisanteries : moqueur, Boogie tente de convaincre Barney d'aller nager avec lui, puis plonge dans le lac et disparaît à jamais.

La deuxième version de la scène, relatée vers la fin du roman, diffère à bien des égards du premier récit. Tragique et cruelle, elle donne lieu à un échange de dures accusations.

- You were once a writer and a damn good one, but now you're just another druggie with pretensions.

- I've failed in my duty to you. I was supposed to amaze the world so that one day you could brag "If not for my help".

- You're pathetic.

- Oh no. I'll tell you what's pathetic. Pathetic is a man so empty that he needs somebody else's achievements to justify his own life. ${ }^{24}$ (1997, p. 383)

\footnotetext{
${ }^{23}$ Je traduis : « Une lettre pouvait arriver de Cuba, adressée à Boogie [...], et elle avait été écrite par Ernest Hemingway. »

${ }^{24}$ Je traduis : «Tu étais jadis un écrivain, et un excellent. Mais tu n'es désormais qu'un autre drogué avec des prétentions. — «J'ai échoué, je n'ai pas su respecter les devoirs qui m'incombaient. Je devais amuser la galerie afin que tu puisses un jour dire "sans mon aide..." »— « Tu es pathétique. »— « Oh non. Je
} 
M.E. LAPOINIE, «Jake, Joshua et Bamey, intellectuels et transfuges: le paradoxal héroïsme des personnages de M. Richler», dans Y.HAMEl et M. BOUCHARD(dir),Portraitdel'homme de lettres en héros, @nalyses, hiver2006

Boogie, apprend-on alors, a accepté le soutien financier d'un ami prospère qu'il méprisait. Prometteur certes, il a perdu en cours de route cette souveraineté, cette liberté tant prisées par Barney — la dépendance de Boogie aux drogues accentue d'ailleurs cette idée de la perte de soi. Aussi l'œuvre de Boogie, demeurée fragmentaire et inachevée, ne témoigne-t-elle que de ses prétentions démesurées. Le modèle, la figure tutélaire, sont ici déboulonnés: celui qui se prétendait engagé dans une pure démarche artistique, celui qui se disait dépourvu de désirs matériels, s'est parjuré, s'est révélé encore plus faible que son mécène.

Et ce renversement, loin d'être anodin, me semble révélateur d'une prise de conscience qui traverse, de manière parfois moins explicite, l'œuvre entière de Richler. Par ce procès des prétentions de l'écrivain - auquel est aussi soumis le très institutionnel Terry McIver —, Richler confère une fin logique à une conception de l'héroïsme qui semble de prime abord antithétique et paradoxale. Jake, Joshua et Barney ont-ils réellement abdiqué leurs idéaux de jeunesse? Ont-ils vraiment renoncé à leurs principes, à leur vision rêvée de l'art et de la société? Ou ont-ils plutôt compris, au fil de parcours parfois tortueux, qu'à force de projeter leurs ambitions sur les autres - qu'il s'agisse d'un cavalier introuvable, d'un vilain docteur allemand ou d'un ami écrivain idéalisé —, ils avaient évité de réfléchir sur leur propre engagement? Qu'ils avaient refusé d'occuper leurs propres places, de jouer leurs propres rôles? Qu'ils avaient, en somme, prétendu être. Or, comme le révèle l'exemple de Barney's Version, la prétention est une forme de trahison, de méconnaissance de soi et de ses capacités réelles. Prétendre, c'est jouer à être autre... C'est miser sur ce qui nous fait défaut plutôt que sur ce que l'on possède. En reconnaissant la vanité de certains idéaux, peut-être se rapproche-t-on, tout compte fait, de la véritable bravoure... En d'autres mots, Hemingway seul peut vraiment être Hemingway.

vais te dire ce qui est pathétique. Pathétique est l'homme si vide qu'il a besoin des réussites d'autrui pour justifier sa propre existence. » 
M.E. LAPOINIE, «Jake, Joshua et Bamey, intellectuels et transfuges: le paradoxal héroïsme des personnages de M.Richler», dans Y.HAMElet M.BOUCHARD(dir),Portraitdel'homme de lettres en héros, @nalyses, hiver2006

\section{Bibliographie}

Adorno, Theodor. 1991 [1951], Minima Moralia. Réflexions sur la vie mutilée, traduit de l'allemand par Eliane Kaufholz et Jean-René Ladmiral, Paris, Payot, coll. « Critique de la politique ».

DAUNAIS, Isabelle. 2002, Frontière du roman. Le personnage réaliste et ses fictions, Montréal, Presses de l'Université de Montréal / Presses universitaires de Vincennes, coll. « Espace littéraire ».

GreEnSTEIN, Michael. 1989, Third Solitudes: Tradition and Discontinuity in Jewish-Canadian Literature, Montreal \& Kingston, McGill-Queen's University Press.

Nepveu, Pierre. 2004, Lectures des lieux, Montréal, Boréal, coll. «Papiers collés».

Ravvin, Norman. 1997, A House of Words. Jewish Writing, Identity, and Memory, Montreal \& Kingston, McGill-Queen's University Press.

RICHLER, Mordecai. 1998 [1997], Barney's Version, London, Vintage.

—. 1990, Broadsides. Reviews and Opinions, Toronto, Viking.

-. 2001, Joshua Then and Now, Toronto, McClelland \& Stewart.

—. 1972, Shovelling Trouble, Toronto, McClelland and Stewart.

—. 1989 [1971], St. Urbain's Horseman, Toronto, McClelland \& Stewart, Series: « New Canadian Library ».

SHEPS, David. 1974, «Waiting for Joey: the theme of the vicarious in St-Urbain's Horseman », Journal of Canadian Fiction, Vol. III, No. 21, December, p. 83-94.

WARREN, Paul. 1989, Le Secret du star-system américain. Une stratégie du regard, Montréal, L’Hexagone. 